\title{
Implications of CSF3R and other Novel Mutations in Chronic Neutrophilic Leukemia
}

\section{Villatoro TM and Uppal GK*}

Department of Pathology, Anatomy and Cell biology,

Thomas J efferson University, USA

*Corresponding author: Uppal GK, Department of Pathology, Anatomy and Cell biology, Thomas J efferson University, 117 South $11^{\text {th }}$ Street, Suite 301, Philadelphia, PA, USA

Received: February 17, 2017; Accepted: February 20, 2017; Published: February 23, 2017

\section{Editorial}

Chronic Neutrophilic Leukemia (CNL) is a rare but potentially aggressive type of Myeloproliferative Neoplasm (MPN) [1]. According to the current 2008 World Health Organization (WHO) diagnostic criteria, CNL is characterized by peripheral blood leukocytosis of $\geq 25$ $x 1^{10}$ /L ( $80 \%$ of which are mature neutrophils and less than $10 \%$ are immature granulocytes); hepatosplenomegaly; a hypercellular bone marrow with myeloid hyperplasia, normal myeloid maturation and $<5 \%$ blasts (less than $1 \%$ in the peripheral blood) [1]. Molecular and cytogenetic analysis must be performed to confirm the absence of Philadelphia chromosome with translocation $\mathrm{t}(9 ; 22)$ (BCR-ABL1), and rearrangements in genes encoding platelet derived growth factor receptors alpha and beta $(P D G F R A / B)$ and fibroblast growth factor receptor 1 (FGFR1) [1]. CNL is essentially a diagnosis of exclusion among the MPNs. Additional molecular analyses in the pathogenesis of CNL have been recently studied to better specify the diagnostic criteria of CNL and propose potential therapeutic targets.

Recent studies have determined that a particular mutation in the colony stimulating factor 3 receptor (CSF3R) may play a role in the development of CNL [2]. Germline CSF3R mutations have been previously reported in more than $30 \%$ of patients with Severe Congenital Neutropenia (SCN) [3]. The CSF3R gene is located on the short arm of chromosome 1. The gene encodes the transmembrane receptor for granulocyte colony-stimulating factor (G-CSF), which is involved in the proliferation, differentiation and function of granulocytes $[2,3]$. CSF3R signals through a number of pathways including the JAK-STAT pathway and the SRC tyrosine kinase pathway. Mutations that alter the receptor structurally and functionally disrupt its ability to regulate granulocytic differentiation and increase granulocytic proliferative capacity [2].

In a seminal study, Maxson et al. reported the usefulness of CSF3R mutation for the diagnosis of CNL [2]. In 27 patients with CNL and aCML, the CSFR3 mutation was identified in 16 patients (59\%). 90\% of the patients with CNL contained a CSF3R mutation, whereas only $45 \%$ of patients with aCML contained a mutation. The most common mutations were detected in the membrane proximal region and include T618I (consists of a single base substitution of C-to-T at nucleotide 1853 on exon 14) and T615A. Other mutations that were noted include frame shift and nonsense mutations in the cytoplasmic tail that lead to truncation. Through drug sensitivity profiling, it was observed that mutations that lead to truncation of the cytoplasmic tail lead to dysregulation in the SRC family-TNK2 kinases, whereas mutations in membrane proximal lead to dysregulation in JAK family kinases. An additional finding via in vitro drug sensitivity assays was the overall clinical improvement noted in patients with CNL that had CSF3R T681I who were treated with ruxolitinib, a JAK1/2 inhibitor, whereas those who had truncated mutations responded selectively to dasatinib, a SRC kinase inhibitor. This observation supported the theory that patients with CNL may show differences in sensitivity to different pathway inhibitors based on the type of CSF3R mutation they harbored.

Pardanani et al. further validated the previous findings by sequencing exons 14-17 of CSF3R in clinical cases suggestive of CNL or aCML [4]. Out of 54 total cases, 12 cases met WHOdefined criteria for CNL and 9 cases were WHO-defined aCML [3]. An overall mutational frequency of $100 \%$ was observed in WHOdefined CNL cases, in contrast to $0 \%$ seen in WHO-defined aCML. The primary mutation encountered was CSF3R T618I $(\mathrm{n}=10)$. They also reported SETBP1 mutations in 33\% patients. Another update of this series has reported SETBP1 mutation in $40 \%$ in CSF3R mutated CNL [5]. In another study, a group of 14 CSF3R mutated CNL patients were screened for additional mutations [6]. The most frequent co-mutation was ASXL1 (8 patients; 57\%). Fivepatients' harbored SETBP1 mutations (38\%), 2 patients had both mutations. Two patients with SETBP1 (not ASXL1) mutations developed blastic transformation. Two patients evolved into Chronic Myelomonocytic Leukemia (CMML); both these patient had ASXL1 mutations but wild type SETBP1 gene. On a multivariate analysis, ASXL1 mutation and thrombocytopenia were reported to be independent risk factors for survival. Other rarely reported mutations in CNL include JAK2 V617F, and CALR.

Since the discovery of CSF3R mutations in CNL, several cases of CSF3R mutated CNL and aCML have reported that received Ruxolitinib. A patient carrying CSF3R T618I was reported to have dose dependent reduction in neutrophil count and resolution of thrombocytopenia [7]. At present, ruxolitnib remains a second line treatment in patients who fail hydroxyurea [8].

Two cases of CSF3R and SETBP1 mutated CNL have been reported so far that failed to respond to ruxolitnib (JAK1/2 inhibitor). This raises the possibility of ineffectiveness of JAK inhibitors in CSF3R mutated CNL cases harboring SETBP1 mutation [5].

These findings, as well as other gene mutations recently found to be implicated in CNL, such as mutations in set binding protein 1 (SETBP1) and JAK2, have culminated in a revision of the current WHO diagnostic classification of CNL to include the molecular 
criterion [9]. Presence of CSF3R T618I or other activating mutations is one of the diagnostic criteria in the current updated WHO 2016 classification of hematopoietic neoplasms. In the in the absence of CSF3R mutation, persistent neutrophilia, splenomegaly and no identifiable cause of reactive neutrophilia including plasma cell neoplasm, should be demonstrated. In presence of any these confounding factors, clonality of myeloid cells must be demonstrated by cytogenetic or molecular studies [9].

With the recent discovery of CSF3R mutations, CNL no longer remains a diagnosis of exclusion. Presence of CSF3R and /or other recurrent mutations makes its distinction easier from reactive leukocytosis. This has also led to better characterization of the disease and easier differentiation from its close mimicker, atypical chronic myeloid leukemia. The CSF3R mutations have proven potential to not only the diagnosis of CNL but to the development of possible target therapies also. SETBP1 and ASXL1 mutations appear to be of prognostic significance and correlate with disease progression.

\section{References}

1. Bain BJ, Brunning RD, Vardiman JW, Thiele J, Swerdlow SH, Campo E, et al. Chronic neutrophilic leukaemia WHO Classification of Tumors of Haematopoietic and Lymphoid Tissues. Lyon, France: IARC Press. 2008 38-39
2. Maxson JE, Gotlib J, Pollyea DA. Oncogenic CSF3R mutations in chronic neutrophilic leukemia and atypical CML. N Engl J Med. 2013; 19: 1781-1790.

3. Klimiankou M, Mellor-Heineke S, Zeidler C. Role of CSF3R mutations in the pathomechanism of congenital neutropenia and secondary acute myeloid leukemia. Ann NY Acad Sci. 2016; 1: 119-125.

4. Pardanani A, Lasho TL, Laborde RR. CSF3R T618I is a highly prevalent and specific mutation in chronic neutrophilic leukemia. Leukemia. 2013; 27: 18701873.

5. Lasho TL, Mims A, Elliott MA. Chronic neutrophilic leukemia with concurrent CSF3R and SETBP1 mutations: Single colony clonality studies, in vitro sensitivity to JAK inhibitors and lack of treatment response to ruxolitinib. Leukemia. 2014; 28: 1363-1365.

6. Elliot MA, Pardanani A, Hanson LA. ASXL1 mutations are frequent and prognostically detrimental in CSF3R-mutated chronic neutrophilic leukemia. Am J Hematol. 2015; 90: 653-656.

7. Stahl M, Xu ML, Steensma DP. Clinical response to ruxolitinib in CSF3R T618-mutated chronic neutrophilic leukemia. Ann Hematol. 2016; 95: 1197.

8. Elliott MA, Tefferi A. Chronic neutrophilic leukemia 2016: Update on diagnosis, molecular genetics, prognosis and management. Am J Hematol. 2016; 913: 341-349.

9. Arber D, Orazi A, Hasserjian R. The 2016 revision to the World Health Organization (WHO) classification of myeloid neoplasms and acute leukemia. Blood. 2016.
Austin J Clin Pathol - Volume 4 Issue 1 - 2017

ISSN : 2381-9170 | www.austinpublishing group.com

Uppal et al. (C) All rights are reserved
Citation: Villatoro TM and Uppal GK. Implications of CSF3R and other Novel Mutations in Chronic Neutrophilic Leukemia. Austin J Clin Pathol. 2017; 4(1): 1047. 